\title{
Casos de éxito en la implementación del mantenimiento predictivo mediante el uso de tecnologías de la industria 4.0 en empresas colombianas
}

\author{
Leidy Marcela Dueñas-Ramírez \\ lmduenasr@eafit.edu.co / Universidad EAFIT \\ Gustavo Adolfo Villegas-López \\ gvillega@eafit.edu.co / Universidad EAFIT \\ Sebastián Castiblanco-Tique \\ scastiblat@eafit.edu.co / Universidad EAFIT \\ Carlos Andrés Castaño-Restrepo \\ cacastanor@eafit.edu.co / Universidad EAFIT
}

Recepción: 31/8/2020 Aceptación: 23/10/2020

Resumen. Con la llegada de nuevas tecnologías el mantenimiento ha dado pasos sustanciales en estas últimas décadas, dejando de lado la toma de datos manuales. Se han adoptado sistemas automatizados y computarizados más eficientes y confiables, técnicas de inspección predictivas y sistemas de control en tiempo real a distancia. Esto ha permitido a las empresas desarrollar planes específicos de mantenimiento a partir del análisis del flujo de datos recolectado, generando beneficios en términos de tiempo y costo. Este artículo expondrá los resultados obtenidos por dos empresas colombianas de gran relevancia en el país en la implementación de tecnologías de la industria 4.0 en los procesos de mantenimiento predictivo. Se hizo un análisis de sus condiciones de éxito y cómo esta metodología ha impulsado su reconocimiento y prestigio en sus respectivos sectores de la industria. Todo este proceso de investigación se lleva a cabo mediante el uso de herramientas y escenarios virtuales, dada la contingencia del COVID-19.

Palabras Clave: industria 4.0 / tecnologías 4.0 / mantenimiento predictivo / eficiencia energética / proceso sostenible / gestión de mantenimiento 


\title{
Success Stories Concerning the Implementation of Predictive Maintenance Through the Use of Industry 4.0 Technologies in Colombian Companies
}

\begin{abstract}
Aвstract. With the arrival of new technologies, maintenance has taken substantial steps in recent decades, leaving aside the manual collection of data. More efficient and reliable automated and computerized systems, predictive inspection techniques and real-time remote-controlled systems have been adopted. This has allowed companies to develop specific maintenance plans based on the analysis of the collected data flow, generating benefits in terms of time and cost. This article will present the results obtained by two top Colombian companies in the implementation of Industry 4.0 technologies in predictive maintenance processes. An analysis of their conditions of success and the way this methodology promoted their recognition and prestige in their respective industry sectors was performed. The entire research process was carried out using virtual tools and scenarios given the current situation with COVID-19.
\end{abstract}

KEYWORDS: Industry 4.0 / technologies 4.0 / predictive maintenance / energy efficiency / sustainable process / maintenance management 


\section{INTRODUCCIÓN}

El término industria 4.0 hace referencia a la cuarta revolución industrial que se entiende como un avance progresivo y contundente dentro de una organización en una cultura de gestión de activos. Todo ello enfocado a las necesidades específicas que tiene cada una de las empresas en los diferentes tipos de industrias (Russmann, Lorenz, Gerbert, Waldner, Justus, Engel y Harnisch, 2015). En Colombia se han obtenido avances considerables en el conocimiento sobre la industria 4.0, este ha aumentado de un 44,5\% en los últimos tres años hasta llegar a un $88,2 \%$ de las empresas del país (ANDI, 2019). Estos datos son alentadores, ya que demuestran el creciente interés hacia el uso de nuevas tecnologías en los procesos y servicios de estas.

La evolución del mantenimiento se ha visto permeada por el desarrollo de estas tecnologías y se ha logrado clasificar desde varios enfoques, entre los que se encuentran el correctivo, que se fundamenta en usar cada equipo hasta la falla catastrófica (Kobbacy y Prabhakar Murty, 2006), y el mantenimiento preventivo, que se ejecuta durante períodos de tiempos específicos y determinados, bajo criterios de profesionales expertos, con el objetivo de minimizar la falla catastrófica y alargar la vida útil de los equipos (Alsyouf, 2007). Los enfoques de mantenimiento correctivo y predictivo en asociación con las tecnologías de la industria 4.0 permiten la implementación de un enfoque nuevo orientado al mantenimiento predictivo basado en tecnologías 4.0 al servicio de la gestión del mantenimiento (Lee, Lapira, Bagheri y Kao, 2013), trayendo múltiples beneficios como la certeza en la predicción de los momentos en que ocurrirá una falla (Selcuk, 2017), teniendo así una gestión del mantenimiento confiable y eficiente (Montero Jimenez et al., 2020).

La implementación de este tipo de mantenimiento permite a las empresas procesos más eficientes, incrementar el valor agregado en sus productos y servicios, mayor competencia nacional e internacional (Dueñas Ramírez y Villegas López, 2020). En el caso de Colombia, hay una adopción gradual de este enfoque de mantenimiento y las empresas reconocen la importancia de tener un personal capacitado en estos temas, por ello se evidencia la necesidad de estudiar e investigar el mantenimiento predictivo y su relación en el uso e implementación de tecnologías 4.0 en sus procesos. Lo anterior resalta la relevancia del talento humano en la evolución de las organizaciones en términos de transformación digital; en consecuencia, es necesario desarrollar estas habilidades y conocimientos, tales como big data, programación, analítica de datos, entre otros, que propicien un ecosistema que integre personas y máquinas con la capacidad de tomar decisiones eficientes (Sahal, Breslin y Ali, 2020).

En Colombia alrededor del $84 \%$ de las empresas priorizan sus inversiones en temas tecnológicos y sobre todo en tecnologías emergentes. El 57 \% de las empresas prioriza la reducción de costos (ANDI, 2019). En consecuencia: se evidencia la demanda potencial en tecnologías emergentes que procuren alcanzar los objetivos de la organización. A pesar de que son muchas las empresas que poseen conocimientos sobre estas tecnologías, es solo un porcentaje reducido las que las usan. De acuerdo con la Asociación Nacional de Empresarios de Colombia (ANDI, 
2019), una tecnología como la inteligencia artificial (IA) aplicada alrededor del $91,5 \%$ de las empresas de Colombia la conocen, pero no la usan, y es utilizada por solo el 21,4\%. Algunas de las posibles causas de ello son los temas sociales y la falta de presupuesto que engloban la falta de cultura, de visión y el desconocimiento (ANDI, 2019). Al ser un tema de carácter humano, su solución puede partir desde la investigación y divulgación de conocimiento y experiencias de los casos exitosos de las pocas empresas donde la implementación de estas nuevas tecnologías ha traído beneficios. Mediante ello se pueden acortar las brechas existentes de tecnología entre estas empresas y aquellas que todavía no poseen la experiencia ni conocimientos suficientes en la implementación de tecnologías 4.0 (ANDI e iNNpulsa, 2018).

La investigación, de carácter cualitativo, se llevará a cabo haciendo uso de herramientas como la entrevista. Para ello se realiza, inicialmente, una investigación documental con el fin de explorar el conocimiento formal, científico y actualizado a nivel mundial, determinar los datos más relevantes y los conceptos más importantes que comparten un interés en los temas asociados a la industria 4.0 y mantenimiento predictivo, con el fin de construir un cuestionario que ayude a resolver el vacío de conocimiento que existe en las áreas de estudio de esta investigación. Se optó por la elaboración de un guion semiestructurado teniendo en cuenta que estos guiones permiten a las personas entrevistadas más espacio a la hora de responder usando un lenguaje representativo e importante para ellos. Estos guiones también permiten comparar las respuestas y de esta manera resultan los conceptos que se usan para obtener más datos en la investigación (Strauss y Corbin, 2004).

Adicionalmente, todo el desarrollo de la investigación se realizó en entornos virtuales incluyendo las entrevistas por la contingencia por el COVID-19. Lo anterior, más que un obstáculo, fue una oportunidad para ampliar más el perfil de los entrevistados, gracias a la modalidad remota que decantó en una transferencia de información más amplia de las empresas que fueron objeto de estudio (Hernán-García, Lineros-González y Ruiz-Azarola, 2020).

A continuación, se enuncia con profundidad la metodología usada para la presente investigación y la secuencia de pasos desarrollados. Luego, se exponen los resultados individuales y comunes a partir de la información brindada por el personal de las empresas seleccionadas. Por último, se analiza y concluye a partir de esta. Se pretende dar respuesta a la pregunta de investigación buscando las condiciones que llevan a que la implementación del mantenimiento predictivo apoyado en la industria 4.0 sea exitosa.

\section{METODOLOGÍA}

Este estudio tuvo como base un proceso de investigación documental y cualitativa y usó la entrevista semiestructurada como herramienta para la recolección de datos empíricos. Estas entrevistas se realizaron a profesionales expertos en las áreas de mantenimiento que pertenecen a empresas colombianas con gran trayectoria y representativas en cada sector de la industria 
nacional. Estos profesionales son personas con alto poder en la toma de decisiones en el área de mantenimiento de estas empresas. Esta investigación se realizó bajo el modelo de acceso remoto debido a la contingencia por el COVID-19. Se contemplaron los siguientes pasos en el desarrollo de esta investigación:

a. Estudiar las publicaciones formales, científicas y normativas existentes alrededor de las temáticas de la industria 4.0 y el mantenimiento predictivo, con el fin de establecer un punto de referencia entre el avance en estos temas a nivel mundial y el avance de Colombia como país en desarrollo.

b. Planificar una entrevista semiestructurada, por medio de una guía, que consideró el estudio de la literatura del punto anterior y desde la experiencia de los investigadores se prepararon las preguntas de las entrevistas. Estas preguntas fueron abiertas y teniendo en cuenta la información que se requería en el proceso de investigación se realizaron aquellas del tipo de preguntas de experiencia o conducta de los entrevistados, preguntas de opinión y preguntas de conocimiento (Patton, 1990). Adicionalmente, se realizaron preguntas de seguimiento y sondeo para profundizar en los temas estudiados. Estas preguntas estuvieron orientadas al detalle, preguntas de elaboración y preguntas de clarificación (Patton, 1990). Se realizaron en total diez preguntas que no impidieran el flujo de la entrevista y fueron expuestas a los entrevistados de forma clara y neutral manteniendo el orden lógico de la temática estudiada. Estas preguntas fueron probadas y evaluadas por profesores y otros profesionales antes de la realización de las entrevistas de este estudio (Morse, Hupcey, Penrod y Mitcham, 2002).

c. Rastrear y contactar empresas colombianas que estén introduciendo en sus procesos a cualquier nivel tecnologías asociadas a la industria 4.0 en la gestión del mantenimiento, haciendo especial énfasis en el mantenimiento predictivo. Se consideraron empresas que hayan mostrado mejoras importantes en sus procesos con el uso de estas tecnologías.

d. Realizar el análisis del contenido de la información recopilada en las entrevistas codificando los datos obtenidos. En este proceso se identificaron las palabras, frases o conceptos, lo cual permitió identificar y analizar los patrones encontrados (Morse y Field, 1995). En esta etapa se familiarizaron los datos y se organizó la información.

e. Categorizar los datos de la información codificada. Esta categorización agrupó los datos en cuatro categorías que fueron talento humano, cultura organizacional, toma de decisiones basadas en datos y tecnologías 4.0 en el mantenimiento predictivo. Estas categorías se definieron teniendo en cuenta que todos los datos estuvieran incluidos y tienen sentido y representan la información encontrada en la entrevista. Esta definición de categorías fue revisada por profesionales expertos ajenos a la 
investigación, quienes aprobaron la razón por la que fueron definidas. Las categorías fueron válidas según los criterios de homogeneidad interna y externa.

f. Validar los datos y conclusiones obtenidas de la investigación. En primer lugar, la validez interna se determinó debido a que todas las conclusiones a las que se llegaron surgieron de los datos obtenidos. En segundo lugar, la validez externa del estudio se comprobó mediante la generalización de los conceptos obtenidos. Teniendo en cuenta que las categorías definidas pueden ser fácilmente aplicables a otros escenarios en el área de mantenimiento, no solo al mantenimiento predictivo y a contextos donde no se emplee la industria 4.0 en sus procesos (Guba y Lincoln, 1982). Las categorías definidas son semejantes no solo a categorías estudiadas en la gestión de mantenimiento, estas aplican también a otras áreas de estudios administrativos y logísticos.

g. Definir los casos de éxito más representativos encontrados en el proceso e investigación, teniendo en cuenta toda la información codificada y categorizada en el presente estudio.

\section{RESULTADOS}

En esta sección se expone el resultado de las entrevistas realizadas, dentro de las que se destacaron dos empresas de gran relevancia en Colombia. Estas empresas han demostrado avances positivos en la implementación del mantenimiento predictivo mediante el uso de herramientas de la industria 4.0. La primera empresa es el grupo Avidesa Mac Pollo dedicada a productos avícolas, en la que se estudió el área de producción y mantenimiento, tomando el consumo de agua como el caso de estudio. La segunda, del sector educativo, es la Universidad EAFIT, en la que se estudió el área de mantenimiento y el sistema de refrigeración de espacios.

El Grupo Avidesa Mac Pollo nace en 1969 con el objeto social de la distribución de alimentos concentrados para todo tipo de animales. Hoy en día se enfoca en procesamiento de aves, específicamente pollos, y todos los productos que se derivan de esta ave. Esta empresa cuenta con plantas de producción en varios territorios de Colombia y su producción es constante y activa las 24 horas del día. Ha sido reconocida por sus reducciones de emisión de gases invernadero y la sostenibilidad de sus procesos, por la empresa Novozymes. Además, ganó por dos años consecutivos (2015-2016) la calificación como los mejores proveedores de aves para restaurantes según la revista La Barra.

Al ser una empresa del sector alimenticio, el proceso de saneamiento e higiene debe ser estricto, por lo que si no hay un control planeado, los costos pueden incrementarse. Una de las experiencias recientes fue la implementación de un sistema de gestión energética que comprende la inversión en medidores electrónicos de agua con el fin de obtener lecturas por 
zonas de procesos en tiempo real y disponibles en línea. Gracias a la implementación de este sistema en el área de mantenimiento se lograron ahorros mensuales de 5,3\% que corresponden a 14406240 pesos colombianos, durante los años 2018-2019 (Fuentes, 2020). En la figura 1 se puede observar la reducción mensual en consumo de agua en este período de tiempo debido a la implementación de este nuevo sistema. El sistema es una primera incursión de la empresa en tecnologías asociadas a la industria 4.0 mediante un desarrollo propio y que basa su implementación en Python. Este sistema ayuda a graficar y evaluar los datos con respecto a las metas y muestra los incrementos o decrementos de las mediciones con respecto a las metas planteadas por la empresa. También puede identificar de manera rápida las zonas de proceso de producción desfasadas o que generan mayor cambio e impacto. Esto ha permitido un rápido análisis de la información que apoya la toma de decisiones en la recomendación que se le hace a cada proceso dentro del proceso de producción de la empresa, ese mismo algoritmo interactúa con SAP-PM para graficar los indicadores de mantenimiento, costos, producción, entre otros.

Además de ello, estudian de forma constante casos de falla mediante contextos de criticidad y peores escenarios, determinando los elementos críticos con el fin de direccionar el enfoque y mejorar la planeación de mantenimiento. La empresa aspira a dar un paso más en la evolución del mantenimiento predictivo hacia uno de tipo prescriptivo, en donde se usa big data e IA para obtener una solución frente a las fallas que puedan presentar los equipos. Hasta ahora han logrado implementar pruebas piloto, ya que los retos de esta tecnología radican en el manejo del big data, por lo que se deben tener consultorías con empresas externas. Al ser pocas las que prestan el servicio en Colombia, son de alto costo (Fuentes, 2020). Estas pruebas han arrojado buenos resultados, lo que motiva a la empresa a seguir investigando en el mantenimiento predictivo con miras a mejorar procesos y obtener reconocimiento.

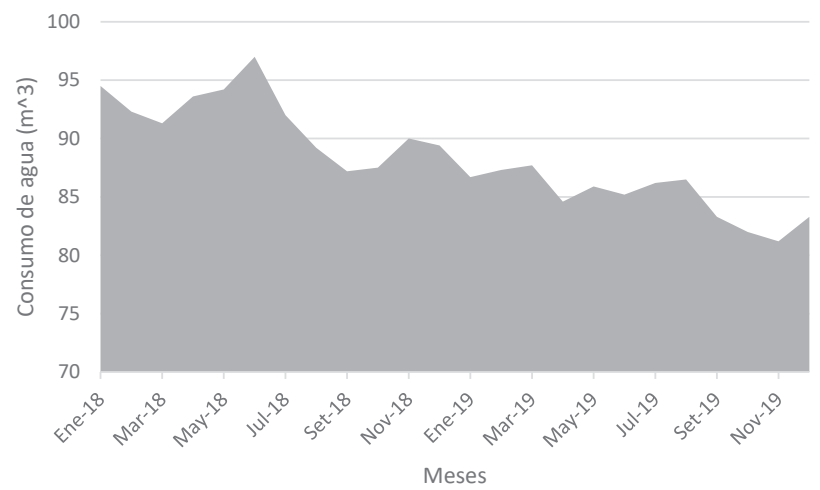

Figura 1. Gasto de agua mensual en producción 2018-2019

Fuente: Fuentes (2020) 
La empresa del sector educativo es la Universidad EAFIT, una de las más importantes del país. Esta institución nace en 1960 como una escuela de administración. Se posiciona hoy en día en el grupo 801-1000 según el QS World University Rankings. Del mismo modo, se destaca por alcanzar el puesto 149 en reputación entre empleadores en el ámbito global, el número de docentes por alumno, y estudiantes y profesores internacionales.

\begin{tabular}{|c|c|c|}
\hline $\begin{array}{l}\text { Entre } \\
\text { fue d } \\
\text { incre } \\
\text { en un }\end{array}$ & $\begin{array}{l}18 \text { y el } 2019 \\
\text { o, teniendo e } \\
\text { ó la capacid } \\
\% \text {. }\end{array}$ & $\begin{array}{l}\text { umento } \\
\text { enta que se } \\
\text { producción }\end{array}$ \\
\hline $\begin{array}{l}\text { La disr } \\
\text { se deb } \\
\text { subest } \\
\text { consur } \\
\text { fue de }\end{array}$ & $\begin{array}{l}\text { hución del valor } \\
\text { a la implement } \\
\text { ión de } 44 \mathrm{kV} \text {; co } \\
\text { del } 2019 \text {, el ah } \\
\text { D } 334225358\end{array}$ & $\begin{array}{l}\text { le } \mathrm{kWh} \\
\text { ión de la } \\
\text { el mismo } \\
\text { rro en el } 2020\end{array}$ \\
\hline $\begin{array}{l}\text { Se esti } \\
\text { será de }\end{array}$ & $\begin{array}{l}\text { a que el consun } \\
\text { n } 52 \% \text { del cons }\end{array}$ & en el 2020 \\
\hline $\begin{array}{l}\text { En dici } \\
\text { aproxir } \\
\text { energía } \\
\text { que so }\end{array}$ & $\begin{array}{l}\text { ibre y enero s } \\
\text { damente, un } 7 \\
\text { e agosto y sep } \\
\text { os de mayor c }\end{array}$ & $\begin{array}{l}\text { onsume, } \\
\% \text { de la } \\
\text { mbre, } \\
\text { sumo. }\end{array}$ \\
\hline Año & Consumo kWh & Costo kWh \\
\hline 2018 & 7664077 & $\$ 351$ \\
\hline 2019 & 8151838 & $\$ 356$ \\
\hline 2020 & 3158480 & $\$ 315$ \\
\hline
\end{tabular}
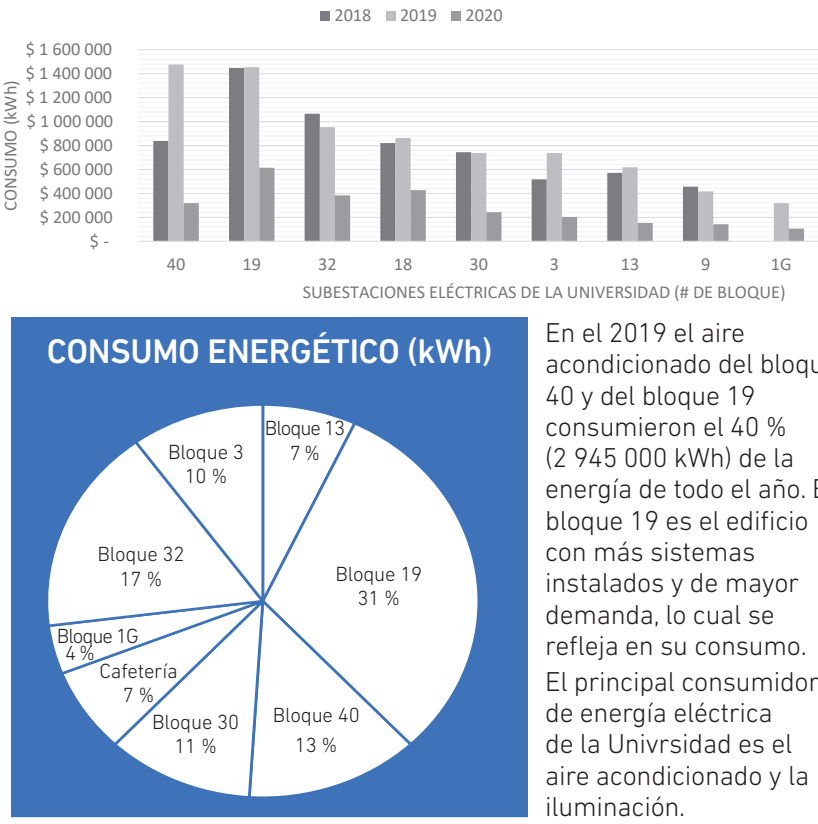

En el 2019 el aire acondicionado del bloque 40 y del bloque 19 consumieron el $40 \%$ (2 $945000 \mathrm{kWh}$ ) de la energía de todo el año. El bloque 19 es el edificio con más sistemas instalados y de mayor demanda, lo cual se refleja en su consumo. El principal consumidor de energía eléctrica de la Univrsidad es el aire acondicionado y la iluminación.

Figura 2. Escenarios de consumo de energía de la Universidad EAFIT

Fuente: Jiménez Arango (2020)

La Universidad EAFIT cuenta con acreditación de alta calidad para la mayoría de sus programas, expedida por el Ministerio de Educación Nacional y la certificación ISO 9001 expedida por el Instituto Colombiano de Normas Técnicas y Certificación (ICONTEC). Cuenta con un aforo de aproximadamente quince mil estudiantes al día y una infraestructura de alrededor de cuarenta edificios. En este caso, el Departamento de Mantenimiento, encargado de todo el sistema de aire acondicionado, fue en el que se centró la investigación. La planta de agua helada tiene una capacidad de operación superior a cualquier otra planta en alguna empresa del sector privado dentro de la ciudad de Medellín, segunda ciudad más poblada de Colombia. El sistema posee un anillo de suministro de agua helada para todos los sistemas de refrigeración de los bloques con una capacidad instalada por encima de la demandada en previsión de construcciones futuras. Este sistema cuenta con un programa de IA alimentado por sensores distribuidos en toda la extensión del anillo y áreas de demanda, que miden en tiempo real las variables críticas en el proceso de refrigeración tales como las variables de caudal, de 
presión de bombas de suministro, eléctricas, temperatura y otros equipos involucrados en el proceso (Jiménez Arango, 2020). La figura 2 describe los escenarios de consumo de energía de la Universidad EAFIT.

En las distintas áreas se obtuvieron reducciones en términos de costo que varían entre el $20 \%$ y $50 \%$, este fue el resultado obtenido de un trabajo realizado a lo largo de seis años en temas asociados a la captura y análisis de datos, lo que generó una cultura de mejora continua en los planes establecidos (Jiménez Arango, 2020). Teniendo en cuenta que la planta de agua helada es el principal consumidor de energía como sistema, se implementa un programa de automatización (CPO), el cual en tiempo real toma decisiones, como lo explica la figura 3:

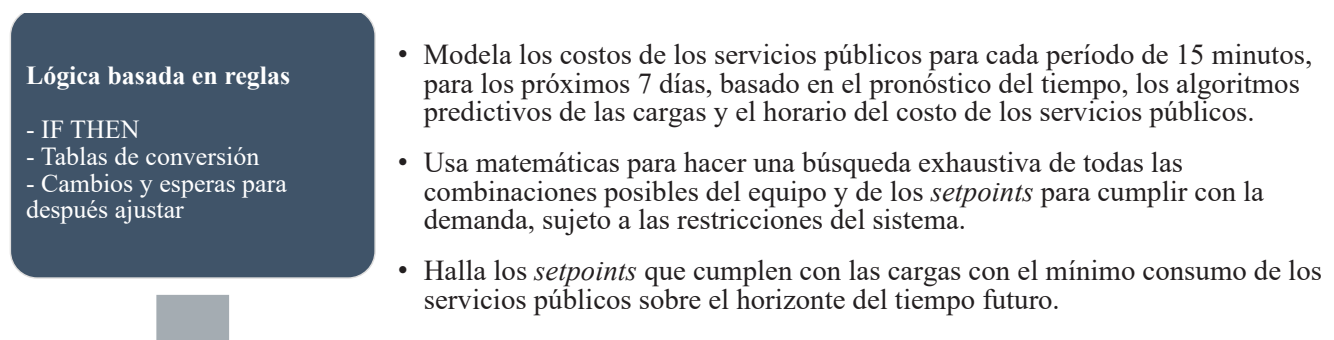

Objective function

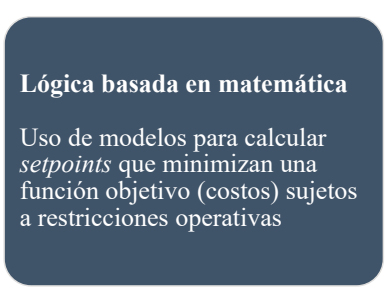

$$
\min (J)=\sum c_{c h} P_{c h \Delta} \Delta t+\sum c_{g a s} f_{g a s} \Delta t+\sum c_{h c} P_{h c}+\sum c_{p m p} P_{p m p}+\sum c_{d e} \max (P)+\sum c_{h r} P_{h r} \Delta t
$$

Equality constraints

$Q_{c h}+Q_{h r}=Q_{\text {Q }}$ oadew_t $t_{0} \pm Q_{-} T E S e w$

$Q_{c h}+Q_{h r}=$ Qloadew_ $t_{1} \pm Q_{-} T E S e w$

$Q_{h c}+Q_{h r}=Q_{\text {loadew_ }} t_{0} \pm Q_{-} T E S$ ew

$Q_{h c}+Q_{h r}=Q_{\text {loadew_t }} t_{0} \pm Q_{-} T E S e w$

\section{Inequality constraints}

$$
\begin{aligned}
Q_{c h} & \leq \max \left(Q_{c h}\right) \\
Q_{T E S} & \leq \max \left(Q_{T E S}\right) \\
Q_{T E S} & \leq \min \left(Q_{T E S}\right)
\end{aligned}
$$

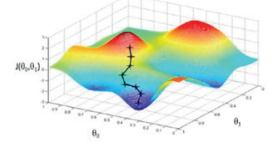

Figura 3. Programa de Automatización CPO, Universidad EAFIT

Fuente: Jiménez Arango (2020)

Con esta herramienta se espera tener ahorros de hasta un $50 \%$ en la operación a toda carga de las instalaciones educativas, lo cual se convierte en un sistema de alto potencial de desempeño a un muy bajo costo. En cifras, lo que anteriormente con 500 toneladas de refrigeración costaba en energía 1825000 pesos COP kWh/día, hoy en día con una instalación de 1500 toneladas de refrigeración se tiene un consumo promedio de 4350000 pesos COP $\mathrm{kWh} /$ día.

En la actualidad el sistema se encuentra en fase de adquisición de datos, con el fin de poder llegar a estos porcentajes de ahorro, para lo cual se requieren varios meses de operación en los que el sistema pueda realizar sus predicciones de acuerdo con las cargas consumidas sin sacrificar las necesidades del sistema y el confort en los espacios. A partir de las entrevistas se identifican datos y experiencias compartidas; a continuación, se presenta esta información 
conjunta englobada por temáticas. Se lograron evidenciar características y comportamientos relacionados en cuanto a la importancia y la medición de variables como base fundamental para la creación de planes de mejora. Se deben establecer las variables más críticas de cada uno de los procesos por analizar con el fin de asignar niveles de criticidad e importancia frente a la magnitud del mantenimiento requerido.

Las nuevas tecnologías, especialmente las pertenecientes a la industria 4.0, juegan un papel fundamental en el mantenimiento predictivo debido a que las técnicas asociadas a este tipo de mantenimiento, de las cuales se destacan el análisis de vibraciones, termografías, entre otras, emplean estas tecnologías en su ejecución, permitiendo un estudio más amplio y riguroso a los equipos. Es de suma importancia que estas técnicas de inspección y medición de variables se encuentren normalizadas, es decir que se rijan mediante normativas o estándares académicamente aceptados. Además, usando sensores y herramientas que integren Internet of Things (IoT) se da paso a sistemas de monitoreo y control en tiempo real, lo que significa una mayor eficiencia en los tiempos de respuesta y la medición de variables. Si las anteriores tecnologías y procesos se realizan de una forma constante en el tiempo, se da la posibilidad de la generación de modelos y algoritmos matemáticos que con ayuda de la IA y machine learning permiten predecir con mayor confiabilidad el comportamiento de los equipos en el desempeño de tareas.

Toda esta recopilación de datos e información, en conjunto con una inversión inicial en personal calificado y en nuevas tecnologías, permite la creación de planes de mantenimiento predictivo confiables que ayudan a reducir costos tanto en reparaciones como en paros de producción, ya que al ser empresas con ciclos de funcionamiento continuo no se pueden permitir tener inconvenientes que afecten su labor normal, de aquí la importancia de los planes de mantenimiento predictivo que logren anticipar los fallos para corregirlos eficazmente sin afectar la producción. También se logran ahorros en gastos de mantenimiento de equipos de poca criticidad con un mínimo de fallas en su ciclo de vida. Si los ahorros se mantienen, con el paso del tiempo se alcanzará un retorno de la inversión inicial y en este punto habrá ganancias para la empresa, lo que demuestra así la importancia de la inversión de capital en mantenimiento predictivo y las tecnologías 4.0 asociadas a este.

Luego de tener formulados los planes de mantenimiento para cada equipo, es importante una correcta ejecución y control sobre este; acá juegan un papel muy importante las tecnologías como IoT o systems, applications, products and data processing (SAP) por la necesidad creciente de medir variables en tiempo real y su almacenamiento para posteriores mejoras de la máquina o proceso. Al ser un flujo de datos tan alto, estas aplicaciones son robustas y se adaptan a la gran cantidad de datos procesados. Como última instancia se verifica el éxito de los planes mediante la formulación de indicadores adecuados a los requerimientos de cada empresa, en los que normalmente se relacionan los productos o principales servicios con todos los costos relacionados a una unidad fija que pertenece a este servicio. 
Por ejemplo, el costo de mantenimiento por kilogramo de alimento procesado en el proceso de producción de la empresa avícola o el costo por cada tonelada refrigerada, donde se busca constantemente su reducción mediante planes de mejora continua.

\section{CONCLUSIONES}

Se identifica que la posibilidad de toma de datos permite la mejora de procesos y el desempeño de equipos; por ello, la determinación de las variables más críticas de dichos procesos es el primer paso en busca de generar planes de mantenimiento predictivo.

El mantenimiento predictivo y el control de este conlleva una serie de costos e inversión inicial, por lo que, si el plan es formulado y ejecutado de forma correcta, se podrá obtener el retorno de la inversión y la reducción de los costos fijos relacionados con el mantenimiento que se solían tener.

Adicional a los costos de reparaciones y mantenimientos, también se pueden evidenciar reducciones en la disminución de los tiempos de parada de producción, ya que se solucionan problemas que se prevé que fallarán en tiempo futuro, eliminando así los riesgos de detención de la producción y su factor de impredecibilidad.

Situaciones como la pandemia mundial vivida en el año 2020 demuestran la necesidad que tienen las empresas de tener sistemas de monitoreo y control a distancia, ya que permiten mantener la actividad productiva sin mayor logística; además, es una forma sencilla de conocer el estado real de cada uno de los equipos de una forma totalmente remota y así ejecutar una toma de decisiones más confiable.

El avance de este tipo de tecnología todavía es incipiente en Colombia, por lo que se ve la necesidad de generar más investigación, proyectos, alianzas empresa-academia con las que las corporaciones podrían acceder a este tipo de avances e implementación de tecnologías con menores costos de inversión y, a su vez, permitiría a los estudiantes adquirir conocimientos mediante su investigación.

Se evidencia que al determinar de forma correcta cada variable crítica del proceso, mediante la realización de un análisis de datos, se obtienen resultados confiables. La estructuración y creación de planes de mantenimiento basados en las necesidades del proceso y el riguroso control de toda la ejecución del proceso facilitaron a las empresas Avidesa Mac Pollo y Universidad EAFIT ser exitosas en la implementación de sus proyectos de innovación en el área de mantenimiento con el uso de tecnologías de la industria 4.0. 


\section{REFERENCIAS}

Alsyouf, I. (2007). The Role of Maintenance in Improving Companies' Productivity and Profitability. International Journal of Production Economics, 105, 70-78. https://doi. org/10.1016/j.ijpe.2004.06.057

Asociación Nacional de Empresarios de Colombia. (2019). Informe de la encuesta de transformación digital 2019. http://www.andi.com.co/Uploads/ANALISIS\%20 -\%20ENCUESTA\%20DE\%20TRANSFORMACI\%C3\%93N\%20DIGITAL\%20 2019\%20-\%20ANDI.pdf

Asociación Nacional de Empresarios de Colombia e iNNpulsa. (2018). Cierre de brechas de innovacióny tecnología. https://www.innpulsacolombia.com/es/cierre-de-brechas-deinnovacion-y-tecnologia

Dueñas Ramírez, L. M., y Villegas López, G. A. (2020). Technological Advances in Computer Science that Define Maintenance Concerns in Industry 4.0 in Colombia. Journal of Physics: Conference Series, 1513, 12010. https://doi. org/10.1088/1742-6596/1513/1/012010

Fuentes, D. (29 de mayo del 2020). Mantenimiento centrado en confiabilidad Grupo Avidesa Mac Pollo. (C. A. Castaño Restrepo, entrevistador).

Guba, E. G., y Lincoln, Y. S. (1982). Epistemological and Methodological Bases of Naturalistic Inquiry. ECTJ, 30(4), 233-252.

Hernán-García, M., Lineros-González, C., y Ruiz-Azarola, A. (2020). Cómo adaptar una investigación cualitativa a contextos de confinamiento. Gaceta Sanitaria. https://doi. org/https://doi.org/10.1016/j.gaceta.2020.06.007

Jiménez Arango, C. A. (15 de mayo del 2020). Entrevista Departamento Mantenimiento y Sistemas de Refrigeración EAFIT. (S. Castiblanco Tique, entrevistador).

Kobbacy, K., y Prabhakar Murty, D. (2006). Springer Series in Reliability Engineering. Springer. https://doi.org/10.1007/978-1-4471-4588-2

Lee, J., Lapira, E., Bagheri, B., y Kao, H. (2013). Recent Advances and Trends in Predictive Manufacturing Systems in Big Data Environment. Manufacturing Letters, 1(1), 38-41. https://doi.org/https://doi.org/10.1016/j.mfglet.2013.09.005

Montero Jimenez, J., Schwartz, S., Vingerhoeds, R., Grabot, B., y Salaün, M. (2020). Towards Multi-model Approaches to Predictive Maintenance: A Systematic Literature Survey on Diagnostics and Prognostics. Journal of Manufacturing Systems, 56, 539-557. https://doi.org/10.1016/j.jmsy.2020.07.008 
Morse, J. M., y Field, P. A. (1995). Qualitative Research Methods for Health Professionals. Sage Publications, Inc.

Morse, J. M., Hupcey, J. E., Penrod, J., y Mitcham, C. (2002). Integrating Concepts for the Development of Qualitatively-Derived Theory. Research and Theory for Nursing Practice, 16(1), 5-18.

Patton, M. Q. (1990). Qualitative Evaluation and Research Methods (2. ${ }^{a}$ ed.). Sage Publications, Inc.

Russmann, M., Lorenz, M., Gerbert, P., Waldner, M., Justus, J., Engel, P., y Harnisch, M. (2015). Industry 4.0: The future of productivity and growth in manufacturing industries. Boston Consulting Group, 9(1), 54-89.

Sahal, R., Breslin, J. G., y Ali, M. I. (2020). Big Data and Stream Processing Platforms for Industry 4.0 Requirements Mapping for a Predictive Maintenance Use Case. Journal of Manufacturing Systems, 54, 138-151. https://doi.org/https://doi.org/10.1016/j. jmsy.2019.11.004

Selcuk, S. (2017). Predictive Maintenance, its Implementation and Latest Trends. Proceedings of the Institution of Mechanical Engineers, Part B: Journal of Engineering Manufacture, 231(9), 1670-1679. https://doi.org/10.1177/0954405415601640

Strauss, A., y Corbin, J. (2004). Bases de la investigación cualitativa. Técnicas y procedimientos para desarrollar la teoría fundamentada. En A. Strauss y J. Corbin, Bases de la investigación cualitativa. Técnicas y procedimientos para desarrollar la teoría fundamentada. Universidad de Antioquia. 